\title{
General Psychiatry Psychopathological outcomes and defence mechanisms in clinically healed adults with a paediatric cancer history: an exploratory study
}

\author{
Antonino Petralia, ${ }^{1}$ Emanuele Bisso (1) , ${ }^{1}$ Ilaria Concas, ${ }^{1}$ Antonino Maglitto, ${ }^{1}$ \\ Nunzio Bucolo, ${ }^{1}$ Salvatore Alaimo (D) , ${ }^{2}$ Andrea Di Cataldo (D) , ${ }^{3}$ \\ Maria Salvina Signorelli (D) , ${ }^{1}$ Alfredo Pulvirenti (D) , ${ }^{1,2}$ Eugenio Aguglia (D) ${ }^{1}$
}

To cite: Petralia A,

Bisso E, Concas I, et al. Psychopathological outcomes and defence mechanisms in clinically healed adults with a paediatric cancer history: an exploratory study. General Psychiatry 2021;34:e100307. doi:10.1136/ gpsych-2020-100307

$\mathrm{AP}$ and $\mathrm{EB}$ are joint first authors.

Received 18 June 2020 Accepted 20 May 2021

Check for updates

(c) Author(s) (or their employer(s)) 2021. Re-use permitted under CC BY-NC. No commercial re-use. See rights and permissions. Published by BMJ.

${ }^{1}$ Department of Clinical and Experimental Medicine, Psychiatry Unit, University of Catania, School of Medicine, Catania, Italy

${ }^{2}$ Department of Mathematics and Computer Science, University of Catania, Catania, Italy

${ }^{3}$ Department of Clinical and

Experimental Medicine,

Pediatric Hemato-Oncology Unit, University of Catania, School of Medicine, Catania, Italy

Correspondence to Dr Emanuele Bisso; emanuelebisso@virgilio.it

\section{ABSTRACT}

Background The incidence of paediatric cancers has increased in recent years; however, with advances in the treatment of paediatric cancer, almost $80 \%$ of children and adolescents who receive a diagnosis of cancer become long-term survivors. Given the high stress levels associated with cancer, it becomes important to ascertain the risk and likelihood of psychiatric disorders in adult paediatric cancer survivors.

Aims This study aims to investigate the relationship between defence styles and predisposition to psychiatric diseases in adults with a history of paediatric cancer.

Methods We performed an explorative study on a sample of 66 clinically healed adults with a history of paediatric cancer (survivors) during follow-up visits at the University Hospital 'Policlinico G Rodolico' of Catania (Italy) and 98 healthy controls among medicine students. We administered the Defence Mechanism Inventory (DMI) to assess defence styles. The Symptom Checklist90-Revised (SCL-90-R) and the Davidson Trauma Scale (DTS) were administered to assess psychopathological indices. We conducted comprehensive statistical analysis based on correlation analysis and mediation analysis to investigate the relationship between defence styles and psychopathological outcomes in survivors compared with controls.

Results The survivors obtained statistically significant lower values in TAO, PRO and TAS defence styles and a higher value in REV. Both groups showed non-pathlogical mean scores in DTS and SCL-90-R (with an exception of the obsessive-compulsive subscale), with lower mean values among survivors. The results of mediation analysis showed that TAS had mediation effects on interpersonal sensitivity, anxiety, PSDI, GSI and avoidance, while TAO had mediation effects on DTS total score and intrusivity. Thus, for these psychopathological indices, the effect of the oncological pathology was indirect and mediated by TAO or TAS. Our analysis exlcluded mediation effects between the remaining variables and defence styles.

Conclusion Integrating data from mediation and correlation analysis, we found how the decreasing of TAS utilization in survivors as the consequence of cancer history, has decreased interpersonal sensitivity, anxiety and GSI score in these subjects compared with controls. Similary, the decrease of TAO utilization played a role in lower values of DTS total score and intrusivity subscale. Unexpectedly, our analysis excluded relationships between cancer history, other defence styles and psycopathological scores as we initially assumed.

\section{INTRODUCTION}

Cancer is a debilitating condition that is considered to be one of the major causes of mortality in children in both developed and developing countries. ${ }^{1}$ A significant increase has been observed in the number of diagnosed cases, although the incidence varies considerably between and within the described regions, and by cancer type, sex, age and racial and ethnic group. ${ }^{2}$ As a result of advances in treatment, almost $80 \%$ of the children and adolescents who receive a diagnosis of cancer become long-term survivors $^{3}$; while this figure seems encouraging, there are negative consequences that cannot be ignored, such as the possible increase in psychological sequelae. Receiving a diagnosis of cancer represents a traumatic and stressful event to adapt to, to which patients must react with important life changes. ${ }^{4}$ In this regard, it is interesting to note how the stress component seems to act on the neoplastic disease itself, as it has recently been identified that tumour cells manifest a state similar to stress, which acts on the tumourigenesis. ${ }^{5}$ Multiple studies have been conducted to understand if cancer can be a vulnerability factor for psychiatric conditions, and some of these have shown an increased incidence in psychiatric conditions. According to the data reported in the meta-analysis by Swartzman et $a l^{6}$, cancer survivors seem to have 1.66 times the odds of post-traumatic stress disorder (PTSD) compared with controls who did not have cancer, but it would seem that the prevalence of adjustment disorder among cancer survivors exceeds that of PTSD, with a prevalence 
equal to $12.4 \% .^{7}$ Other studies have found an increase in cognitive fatigue and depressive symptoms in childhood cancer survivors ${ }^{8}$ and a considerable percentage is affected by suicide ideation decades after having survived cancer. ${ }^{9}$ Comparatively, few descriptions exist of positive psychosocial factors after cancer, even though it has been shown that more than $80 \%$ of survivors of childhood cancer report at least one positive consequence of this experience. ${ }^{10}$ Resilience seems to play an increasingly important role in psycho-oncology research, despite the difficulties derived from the various descriptions of it in the literature. According to one of them, resilience is defined as baseline characteristics or traits, which are identified at the time of first interactions and enable individuals to thrive in the face of adversity. ${ }^{11}$ Some examples may include personal attributes such as optimism, hope, motivation and spirituality and pre-existing social support. Other authors suggested that resilience was a trajectory or mechanism of positive adaptation that changeed over time and protected against psychological distress $^{12}$, while a third stream of thought defines resilience as a development process consequent to traumatic stress, which is evidenced by a relatively positive psychosocial functioning. ${ }^{13}$ Such resilience outcomes include posttraumatic growth (PTG) and the absence of psychological distress, and it is considered a resource in coping with psychological distress and traumatic experiences. ${ }^{14}$ Overcoming cancer can be a chance for personal growth, but not everyone reacts to adversities in the same way, which is why it is important to identify which factors discriminate patients with cancer, as well as subjects with a history of cancer who experience psychological growth from those who do not. Defence mechanisms, which were defined by Freud in his theory of psychoanalysis as the unconscious attempts that individuals use to overcome excessive anxiety, also seem to play an important role in adjustment to adverse situations. Immature defence mechanisms are positively correlated with psychopathology measures, while mature defences are correlated with better psychological adjustment. ${ }^{15}$ Although some scientists continue to consider them outdated concepts, recent studies have been conducted to confirm their importance in patients with cancer. ${ }^{16}$ In a recent meta-analysis ${ }^{17}$, a correlation was observed between the defence mechanism of repression and worse physical and emotional health-related quality of life, and the defences of action predicted fewer chances of survival. Further investigations of these mechanisms seem to be fundamental to understand how to manage the psychological recovery of patients with cancer.

\section{Aim of the study}

To date, few studies have been conducted to study the correlation between defence mechanisms and predisposition to psychiatric disease among adult survivors of paediatric cancer. Our paper aims to fill this gap. We conducted an exploratory study on the effects that exposure to the life event represented by paediatric cancer can have, on adults with a history of paediatric neoplasia as

\begin{tabular}{|c|c|c|}
\hline & Survivors & Controls \\
\hline Number of samples (n) & 66 & 98 \\
\hline Men (\%) & $31(47)$ & $49(50)$ \\
\hline Women (\%) & $35(53)$ & $49(50)$ \\
\hline Mean age (SD) & $23.7(5.033)$ & $20.5(1.169)$ \\
\hline $\begin{array}{l}\text { Mean age of diagnosis in } \\
\text { years (SD) }\end{array}$ & $7.93(4.828)$ & - \\
\hline $\begin{array}{l}\text { Mean duration of disease in } \\
\text { months (SD) }\end{array}$ & 22.26 (11.989) & - \\
\hline $\begin{array}{l}\text { Education ( } n \text {, university } \\
\text { degree) }\end{array}$ & 16 & 0 \\
\hline $\begin{array}{l}\text { Education (n. high school } \\
\text { diploma) }\end{array}$ & 50 & 98 \\
\hline $\begin{array}{l}\text { Education (n, elementary } \\
\text { school) }\end{array}$ & 0 & 0 \\
\hline $\begin{array}{l}\text { Occupational status (n, } \\
\text { worker) }\end{array}$ & 30 & 0 \\
\hline $\begin{array}{l}\text { Occupational status (n, } \\
\text { student) }\end{array}$ & 36 & 98 \\
\hline $\begin{array}{l}\text { Occupational status ( } \mathrm{n} \text {, } \\
\text { unemployed) }\end{array}$ & 2 & 0 \\
\hline
\end{tabular}

compared with controls, from a psychopathological point of view. We also want to identify if and how defence mechanisms adopted by subjects can affect the risk of psychiatric diseases in adulthood.

\section{MATERIAL AND METHODS}

From April 2018 to December 2019, we recruited 68 adults, clinically healed, with a history of paediatric cancer (survivors) and 98 healthy controls. A matched group design was conducted using the following inclusion criteria for cases: age $\geq 18$ years, with a history of neoplastic pathology in childhood, clinically healed and no antineoplastic therapy in place. For the control group, the inclusion criteria were as follows: age $\geq 18$ years, with no history of psychiatric disorders and serious medical diseases. No significant difference was reported for sex; on the other hand, age of controls was slightly lower than cases (see table 1). We included all patients who accepted to participate the scheduled post-disease follow-up visits carried out at Pediatric Onco-Haematology Clinic of the University Hospital of Catania (Italy). Two patients from the case group accepted to participate but later refused to complete the assessments, and they were therefore not included in the final analysis, thus the final analysis was conducted on 66 of the 68 recruited subjects. Because it was not feasible to construct a randomised controlled sample from the entire population, we selected young people with the same demographic characteristics of the survivors by sampling from first-year and second-year students of medicine of the University of Catania, coming 


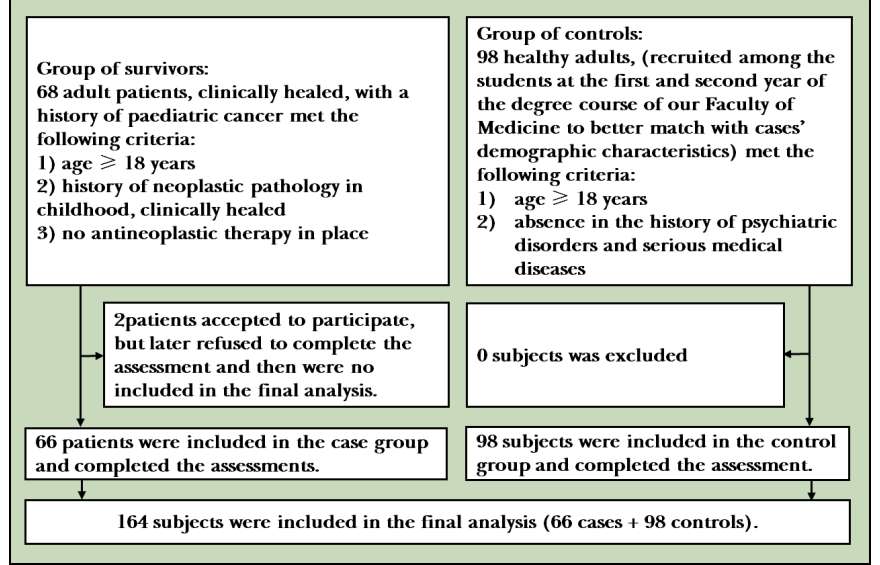

Figure 1 Study flowchart.

from the same geographical area of the survivors. The entire process is summarised in the flowchart in figure 1.

\section{Data collection and assessments}

First, we conducted a brief anamnestic interview among survivors to collect general clinical data and relevant information including sex, age, type of paediatric cancer, age of diagnosis and duration and healing age. For controls, we collected information about sex, age and history of psychiatric and serious medical diseases. For both groups, we also collected data on education and current employment status (working, studying or unemployed). Then psychometric tests were administered to evaluate psychological defence styles, psychopathological indices and eventual post-traumatic stress symptoms. The defence style was assessed through the Defence Mechanism Inventory (DMI).$^{18}$ It showed good validity ${ }^{19}$, measuring five defence styles comprising TAO (turning against objects, that includes identification with the aggressor and displacement), TAS (turning against self), REV (reversal, which includes repression, denial, negation and reactive formation), PRO (projection) and PRN (principalisation, that regards isolation and rationalisation). DMI is a semiprojective test that includes 10 short stories, two for each area of investigation: authority, independence, masculinity or femininity, competition and the area of conflicts arising in situations of daily life. The psychopathological indices have been investigated through self-report Symptom Checklist-90-Revised (SCL-90-R). ${ }^{20}$ In a validation study by Schmitz et $a l^{21}$, the internal consistency, measured by Cronbach's alpha coefficients, was found to be high for the global scale and subscales. According to Schmitz et al, SCL-90-R has proven to be a useful tool for measuring psychological status, measuring changes in outcome studies or screening for mental disorders. ${ }^{21}$ SCL-90-R assesses nine primary symptomatic dimensions: somatisation (SOM), obsessive-compulsive (OBS), interpersonal sensitivity (INT), depression (DEP), anxiety (ANX), hostility (HOS), phobic anxiety (PHOB), paranoid ideation (PAR) and psychoticism (PSY). In addition to these dimensions, there are three global scores referred to as the Global Severity Index
(GSI), the Positive Symptom Distress Index (PSDI) and the Positive Symptom Total (PST). The Davidson Trauma Scale (DTS) ${ }^{22}$ was used to evaluate the presence of posttraumatic symptoms. It is made up of 17 items that correspond to each of the 17 symptoms of DSM-IV. Items 1-4 and 17 refer to intrusive experiences, items 5-11 to avoidance and obtundation and items 12-16 to hypervigilance. For each item, the subject evaluates the frequency and the severity with a scale from 0 to 4 . DTS has been proven to be reliable and valid, showing good concurrent validity. ${ }^{23}$

\section{Statistical analyses}

We estimated the minimum sample size to obtain a power of $80 \%$ at a significance level of 0.05 , assuming an effect size of 0.6 (as the difference on the mean of TAO on the case/control groups), a minimum sample size of 45 patients in either group (survivor vs control group) was established. Continuous data are expressed as mean (SD) when normally distributed and otherwise as median and IQR, while categorical data are expressed as frequencies and percentages. Proportions were compared by $\mathrm{X}^{2}$ test with Yates' correction for continuity or Fisher's exact test, as appropriate; comparison of continuous variables was performed by Student's t-test or permutation test. A $p$ value of $<0.05$ was considered statistically significant. Factors that may potentially influence the primary outcome were individually compared between cases and controls in univariate analyses, using $\mathrm{X}^{2}$ or Fisher's exact tests for categorical variables; for quantitative variables, as distributions were not normal (Shapiro-Wilk test), the Mann-Whitney test was used. We also conducted a Pearson's Correlation analysis (using a p-value cut-off of $\mathrm{p}<0.05$ ), and a mediation analysis. ${ }^{24}$ Mediation analysis has been conducted using the mediation package ${ }^{25}$ within the $\mathrm{R}$ statistical software.

\section{RESULTS}

\section{Characteristics of samples}

The mean age was slightly lower among controls than survivors, which was 23.7 and 20.5 years, respectively (table 1). The distribution by gender was similar with a slight prevalence of women in the group of cases. The most common type of neoplastic disease was acute lymphoblastic leukaemia (ALL) that was prevalent in our sample compared to other neoplastic diseases. More specifically, there were acute lymphoblastic leukaemia (41 cases), acute myeloid leukaemia ( 1 case), Burkitt's lymphoma (1 case), Ewing's sarcoma (1 case), ganglioneuroma (1 case), hepatoblastoma (1 case), Hodgkin's lymphoma (8 cases), non-Hodgkin's lymphoma (2 cases), osteosarcoma ( 1 case), ovarian teratoma ( 1 case), paediatric myofibroblastic tumour (2 cases), rhabdomyosarcoma (1 case), testicular tumour (1 case) and Wilms' tumour (3 cases). Their mean age of diagnosis was 7.93 years, and the mean duration was 22.26 months. Regarding educational level, in the survivor group, 50 subjects had a high school diploma, while 16 had a university degree. In the control 
group, all 98 subjects were university students and therefore in possession of a high school diploma, but not yet a university degree. As for the employment status, in the group of survivors, 30 subjects worked and 36 studied; in the control group, all 98 subjects were university students (table 1). Forty survivors received only chemotherapy; 14 chemotherapy and radiotherapy; 8 surgery plus chemotherapy; 1 chemotherapy, radiotherapy and surgery; 1 chemotherapy, radiotherapy and haematopoietic stemcell transplantation (HSCT); and 1 chemotherapy and HSCT.

\section{Results of data analysis}

Results of statistical analysis are summarised in tables 2-4. The defence mechanisms appeared to be distributed differently between the survivor and control groups. Adults with a history of paediatric cancer obtained statistically significant lower value in TAO, PRO and TAS but higher in REV, than controls according to a Welch's twosample t-test. These results were statistically significant, especially for TAO and PRO $(\mathrm{p}<0.01)$. PRN was found to be similar in the two groups. All SCL-90-R and DTS scales and subscales had lower scores in the case group compared with the control group (table 3). These results were also statistically significant except for SOM, PHOB and PST for SCL-90-R, and hypervigilance for DTS. Both groups obtained a non-pathological mean scoring in DTS and in SCL-90-R subscales. These last subscales appeared all below the cut-off (greater than or equal to 1), with the exception of OBS subscale for controls (table 2). Because all the subjects in the control group were university students, to remove any bias in the comparison, we divided survivors into two groups (students and workers) and analysed the variables of interest to establish any statistically significant difference. Our analysis showed that the two groups were not distinguishable with respect to education. Therefore, such covariate did not influence the comparison with the control group in which all the subjects were students. Groups were also tested for homogeneity by gender (Fisher's exact test, $\mathrm{p}=0.7$ ) and age (Welch's t-test, $\mathrm{p}<0.001$ ). In addition, we performed a mediation analysis ${ }^{24}$ to quantify how much mediatingvariables can influence the transmittance of change from a cause to its effect. A first mediation mediation model was developed to understand if the patient class (history of childhood cancer or not) can directly influence the DTS or SCL-90-R values, or such effect is mediated through a variable of DMI and indeed indirect. The analysis was run only on pairs in which the patient class significantly influences the DMI variable and the SCL-90-R or DTS variables. For each mediation analysis, we computed the average causal mediation effects (ACMEs), average direct effects ( $\mathrm{ADE}$, represented as $\mathrm{C}^{\prime}$ ), and the total effect (direct+indirect, represented as C). The 'Prop. Mediated' in table 4 describes the proportion of the effect of the patient class on the $\mathrm{Y}$ variable that went through the mediator, which was calculated by dividing the ACME through the total effect. Each mediation had been executed with 500 simulations. The results of mediation analysis (reported in table 4 and figure 2) clearly show that TAS had a mediation effect on interpersonal sensitivity $(\mathrm{p}=0.004)$, ANX $(\mathrm{p}<0.001)$, PSDI $(\mathrm{p}=0.015)$, GSI $(\mathrm{p}=0.004)$ and avoidance $(\mathrm{p}=0.004)$, respectively the mediation models $\mathrm{A}, \mathrm{B}, \mathrm{C}, \mathrm{D}, \mathrm{E}$ (figure 2), while TAO had a mediation effect on DTS total score $(p<0.001)$ and intrusivity subscale $(p<0.001)$, as in the mediation models $\mathrm{F}$ and $\mathrm{G}$. Therefore, for such variables, the effect of the oncological pathology was indirect and mediated by TAO and TAS. Our analysis excluded a mediation effect between the remaining variables and defence styles (not reported in table 4 because it was not significant). The Pearson's correlation analysis showed a positive correlation in the survivor group between TAS and, respectively, INT ( $\mathrm{p}=0.024, \mathrm{r}=0.277)$, ANX ( $\mathrm{p}=0.013$, $\mathrm{r}=0.304)$, GSI $(\mathrm{p}=0.035, \mathrm{r}=0.26)$, OBS $(\mathrm{p}=0.032, \mathrm{r}=0.265)$, PSY $(p=0.019, r=0.288)$ and PST $(p=0.044, r=0.249)$. Among them, INT, ANX and GSI corresponded with the result of mediation analysis, and then we can consider the effect of TAS defence style on these variables as mediated by the specific life event 'history of a healed paediatric cancer'. We found lower TAS in survivors than controls, and we therefore conclude that the decreasing TAS utilisation in survivors as the consequence of cancer history had decreased INT, ANX and GSI score in these subjects. In addition, PRN and REV were lower in survivors than controls, and we found a negative correlation by Pearson's analysis between them and HOS $(\mathrm{p}=0.009, \mathrm{r}=-0.319$ for REV-HOS and $\mathrm{p}=0.001, \mathrm{r}=-0.397$ for PRN-HOS, respectively), and between PRN and ANX ( $\mathrm{p}=0.027, \mathrm{r}=-0.272)$. However, these results were not confirmed by the mediation analysis, and although the lower values of PRN and REV in survivors compared with controls resulted in lower values of ANX and HOS, this effect did not seem to have been clearly influenced by the history of cancer. In a similar way, TAO was significantly correlated with SOM $(p=0.044, r=0.204)$ and HOS $(p=0.001, r=0.322)$, but the mediation analysis did not confirm this result as consequences of cancer history. Regarding DTS scoring, TAO was found to be positively correlated with DTS total score $(\mathrm{p}=0.018, \mathrm{r}=0.239)$, intrusivity $(\mathrm{p}=0.001, \mathrm{r}=0.318)$ and hypervigilance $(\mathrm{p}=0.006, \mathrm{r}=0.278)$. The first two variables coincided with what is highlighted by the mediation analysis and we can therefore consider that the TAO style (less used by survivors than controls) affected the DTS score and hypervigilance due to the history of paediatric cancer. PRO showed a relationship with DTS $(\mathrm{p}=0.042, \mathrm{r}=0.251)$ and hypervigilance $(\mathrm{p}=0.028, \mathrm{r}=0.27)$, but not confirmed by mediation analysis. PRN and REV correlated with hypervigilance $(\mathrm{p}=0.05, \mathrm{r}=-0.242$ and $\mathrm{p}=0.03, \mathrm{r}=-0.267$, respectively). In addition, we performed a subclass analysis on psychometric data, matching survivors and controls with the same education and occupation (tables 1-3) in two comparisons (50 survivors vs 98 controls both with high school diploma; and 36 survivors vs 98 controls both students). These results have been reported in tables 2 and 3 and confirmed the same significant differences between survivors and controls. The 


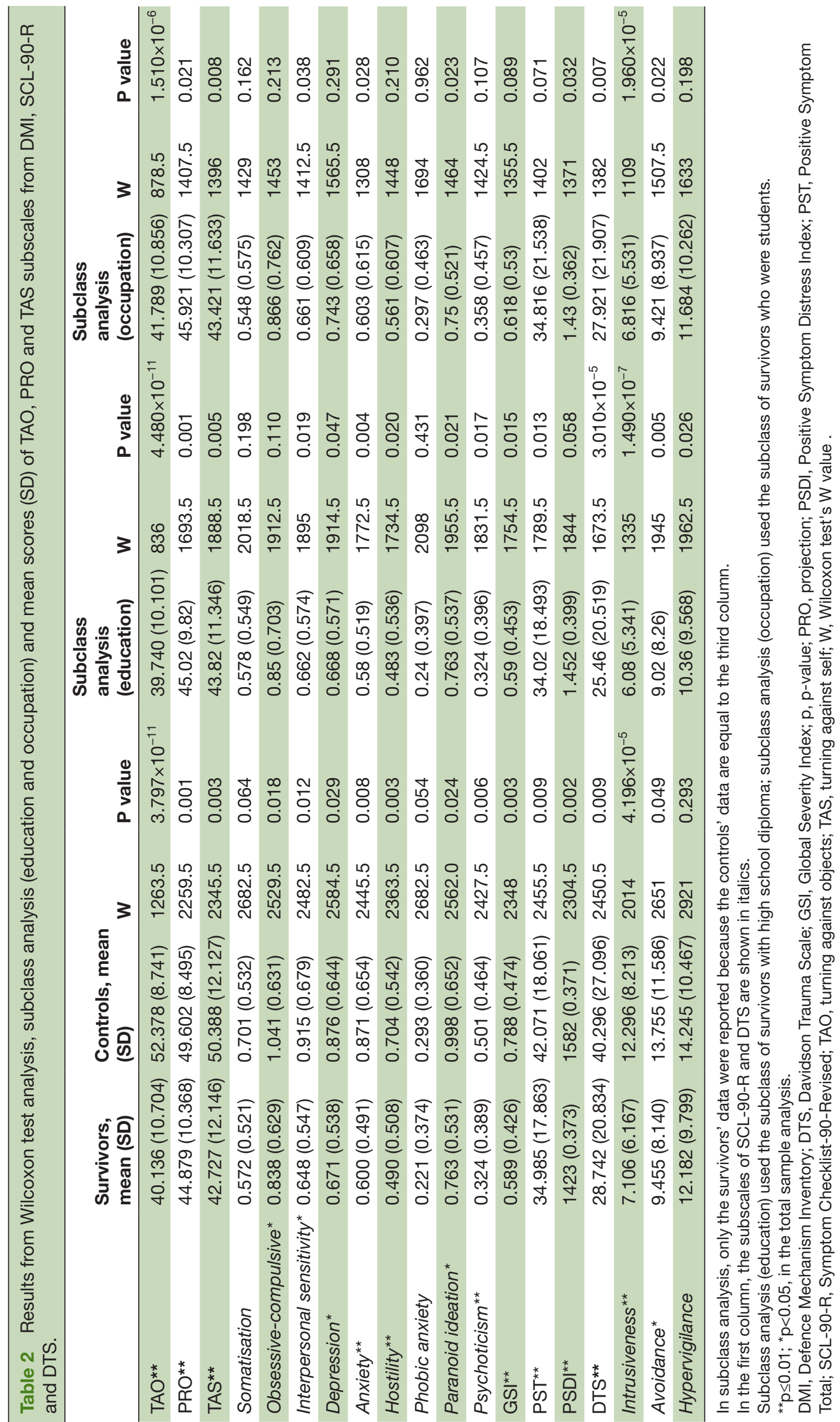




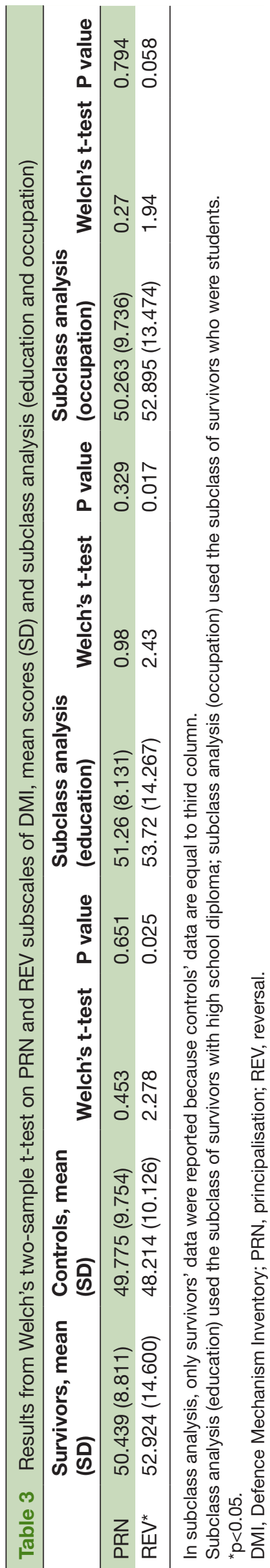

mean scores in subclasses' comparisons were found to be very similar or equal to what was obtained from the analysis on the entire samples.

\section{DISCUSSION}

Main findings

Oncological pathology can have an impact on a person's psychology and psychopathology. A fundamental role in the development of psychopathologies is related to the defence and coping mechanisms that are put in place in response to stressful events. ${ }^{9}$ Two contrasting conditions were described in the literature: according to some studies, cancer could increase individual psychic vulnerability due to traumatic experiences and the consequent physical and psychological sequelae with the onset of PTSD, anxiety disorders and depressive disorders. ${ }^{16} 17$ Other studies instead suggested a protective function of cancer diseases on the future development of mental disorders. ${ }^{14}$ According to our findings, survivor group showed non-pathological scoring in all SCL-90-R subscales, and in comparison with the control group, they showed 30\% lower scores (table 2). This result has statistical significance. In the same way, the survivors achieved lower score in DTS test specifically used to evaluate PTSD symptoms. Moreover, our findings are in agreement with other studies that reported a reduced development of PTSD in subjects with a history of paediatric neoplasm compared with healthy controls, with no history of cancer. ${ }^{12} 13$ DMI showed in survivors lower scores in three of the five defence styles investigated compared with controls: TAO, TAS and PRO. PRN was comparable between the two groups, while REV (including repression, denial and reactive training) was the most used defence mechanism in survivors, more so than controls. These results are in agreement with what was reported in the literature, according to which, repression, understood as the inability to remember or to be aware of desires and feelings, is among the immature defence mechanisms most present in cancer survivors' disturbing thoughts or experiences. ${ }^{15}$ Interesting considerations can be placed on the basis of the higher scores achieved by the survivors with the defence style of REV. This result would make these subjects part of the 'repressors', who would tend to report low levels of mental distress. Therefore, it could be expected that they also reported lower levels of somatic symptoms. However, numerous studies have documented that repressors often show higher levels of autonomic physiological reactivity to stress than non-repressors. Therefore, the survivors falling more frequently in this category could present greater reactivity to stress, but this is to be investigated with further targeted studies. According to some authors, there was a significant association between repression and lower cancer survival $^{26}$, whereas other studies reported that this correlation was minimal. ${ }^{27}$ Another commonly observed immature defence mechanism is denial, with a prevalence of between $4 \%$ and $47 \%$ of subjects with cancer. ${ }^{28}$ Some 
Table 4 Results from mediation analysis: direct and indirect effects of $\mathrm{X}$ variable on $\mathrm{Y}$ mediated by 'mediator' variable

\begin{tabular}{|c|c|c|c|c|c|c|c|c|c|}
\hline$x$ & $\mathbf{Y}$ & Mediator & ACME & $\begin{array}{l}\text { p value } \\
\text { (ACME) }\end{array}$ & ADE & $\begin{array}{l}\text { p value } \\
\text { (ADE) }\end{array}$ & $\begin{array}{l}\text { Prop. } \\
\text { Mediated }\end{array}$ & TE & $p$ value (TE) \\
\hline $\begin{array}{l}\text { Patient } \\
\text { class }\end{array}$ & $\begin{array}{l}\text { Interpersonal } \\
\text { sensitivity }\end{array}$ & TAS & -0.135 & $<0.001$ & -0.132 & 0.124 & 0.506 & -0.267 & 0.004 \\
\hline $\begin{array}{l}\text { Patient } \\
\text { class }\end{array}$ & Anxiety & TAS & -0.105 & $<0.001$ & -0.166 & 0.064 & 0.387 & -0.271 & $<0.001$ \\
\hline $\begin{array}{l}\text { Patient } \\
\text { class }\end{array}$ & GSI & TAS & -0.087 & $<0.001$ & -0.112 & 0.132 & 0.437 & -0.199 & 0.004 \\
\hline $\begin{array}{l}\text { Patient } \\
\text { class }\end{array}$ & PSDI & TAS & -0.065 & $<0.001$ & -0.094 & 0.144 & 0.4070 & -0.159 & 0.016 \\
\hline $\begin{array}{l}\text { Patient } \\
\text { class }\end{array}$ & DTS total score & TAO & -6.377 & 0.008 & -5.177 & 0.264 & 0.552 & -11.553 & $<0.001$ \\
\hline $\begin{array}{l}\text { Patient } \\
\text { class }\end{array}$ & DTS intrusivity & TAO & -2.008 & $<0.001$ & -3.182 & 0.004 & 0.387 & -5.190 & $<0.001$ \\
\hline
\end{tabular}

Please note that only significant mediations were reported $(p<0.05)$.

Prop. Mediated=proportion of effect of the patient's class on the $Y$ variable that goes through the mediator (calculated as ACME/TE).

ACME, average causal mediation effect; ADE, average direct effect; DTS, Davidson Trauma Scale; GSI, Global Severity Index; PSDI, Positive

Symptom Distress Index; TAO, turning against objects; TAS, turning against self; TE, total effect (direct+indirect).

authors believed that minimising or ignoring information relating to the disease was associated with a reduced anxious share and a consequent improvement in quality of life ${ }^{17}$; therefore, denial would be useful in managing stressful situations but only at an early stage, as in the long run, adaptive properties would decline. ${ }^{29}$ On the basis of our results, it seems that the healing process in survivors entails a psychic readjustment of the individual's functionality to consolidate coping skills. This seems to impart more stress resistance to survivors compared with controls, against the onset of psychopathological symptoms. We can interpret these findings as the result of the development of a survivor's greater coping capacity in relation to the oncological disease. The literature confirms such interpretation: a study conducted on the survivors of paediatric carcinoma showed significant psychosocial

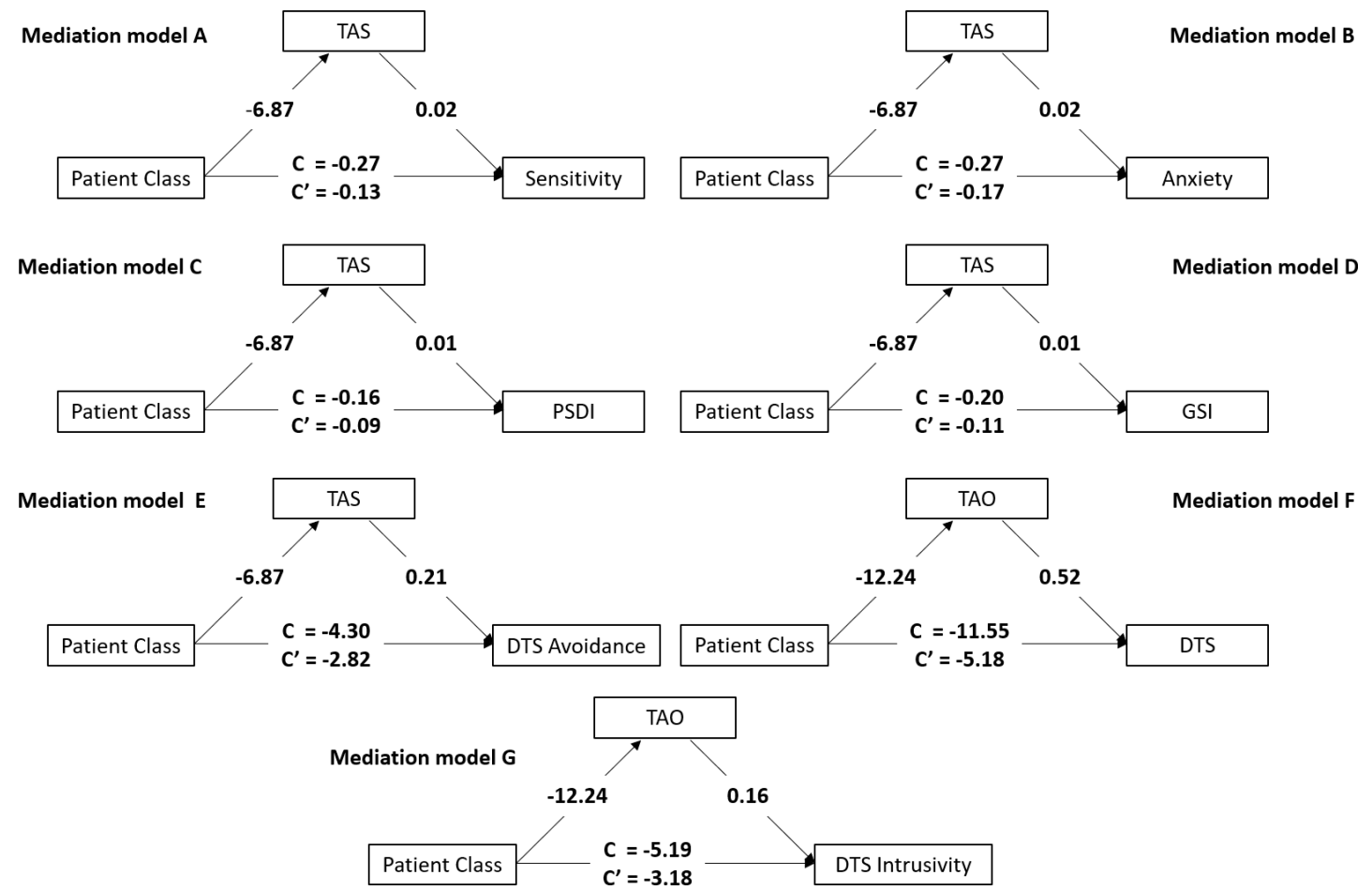

Figure 2 Mediation analysis diagrams. DTS, Davidson Trauma Scale; GSI, Global Severity Index; PSDI, Positive Symptom Distress Index; TAO, turning against objects; TAS, turning against self. 
distress in some subjects, whereas others gained PTG from this experience. PTG is a condition that defines a positive change that occurs as a consequence of particularly stressful events. In other words, the patient achieves a higher level of functioning and well-being than the one before the disease. ${ }^{14} \mathrm{~A}$ mature defence mechanism frequent in patients with cancer seems to be altruism, connected with the sense of gratification of the individual in satisfying the needs of others. ${ }^{15}$ According to some authors, altruism predisposed patients with cancer more to receive support from hospital staff and was negatively associated with depression and anxiety. ${ }^{30}$ It is important to underline that defence mechanisms are related to coping styles ${ }^{31}$, and some studies have shown that immature defence mechanisms are significantly associated with a dysfunctional coping style, such as impotence/despair, which determines a pessimistic attitude that hinders the therapeutic alliance, and reduces the quality of life and survival to cancer. ${ }^{16}$ Some authors have highlighted the importance of the environmental context among the factors capable of inducing PTG, such as access to programmes of psychological and social support during treatment. ${ }^{32}$ The patient goes beyond resilience, with the search for a higher level of functioning and the achievement of even greater well-being than the one preceding the disease. ${ }^{33}$

\section{LIMITATIONS}

A limitation of this study is the lack of a randomisation process in sample selection due to recruitment modalities. Another critical point is that the control group only involved medical students; this could have introduced bias in the comparison, as this group has characteristics, such as, a high level of schooling or the plausible presence of particular personal inclinations that have oriented the choice of this path to graduation. These results should be confirmed on a more heterogeneous and inclusive comparison sample. Furthermore, it would be useful to extend the comparison between survivors and children with cancer to highlight any discrepancies in the defence styles adopted, and to investigate how these can be modelled by oncological pathology.

\section{CONCLUSION}

According to our findings, survivors of childhood cancer showed, compared with controls, lower mean scores in TAO, PRO and TAS defence styles, and higher scores in REV and PRN. The difference of PRN was found to be small and not significant $(p=0.651)$. All the mean scores obtained in the SCL-90-R psychopathological subscales were non-pathological for both groups (with the exception of OBS for controls) and lower in survivors than controls. We believe that the survivors have developed, due to neoplastic pathology, a greater and more evolved coping capacity almost totally independent from the defence mechanisms. In addition, we performed a mediation analysis to evaluate whether there was any association between the defence styles adopted by survivors and the psychopathological indices. Unexpectedly, we found that the only correlation concerned TAS, which has a mediation effect on interpersonal sensitivity, ANX, PSDI, GSI and DTS-avoidance subscale, while TAO had a mediation effect on DTS total score and DTS-intrusivity subscale. Based on our findings, the remaining variables and defence styles were not related to each other as we had initially assumed. Our results, crossing data from mediation and correlation analysis, showed how a decreasing TAS utilisation in survivors, as a consequence of cancer history, has decreased INT, ANX and GSI scores in these subjects compared with controls. In the same way, TAO was found to be positively correlated with DTS total score $(\mathrm{p}=0.018, \mathrm{r}=0.239)$ and intrusivity $(\mathrm{p}=0.001, \mathrm{r}=0.318)$, both in correlation and mediation analyses; therefore, we can conclude that a lower TAO in survivors (considered to be a consequence of history of cancer) leads to a lower score in DTS.

Contributors Study concept and design-AP, ADC, EB and NB. Data collection and assessments-AM, EB, NB and IC. Methodology-AP, APu and EB. Data analysis and interpretation-APu, EB, SA and AM. Drafting of the original manuscript-EB, $I C$ and NB. Manuscript review and editing-EB, APu, IC and NB. Supervision-AP, $\mathrm{APu}, \mathrm{MSS}, \mathrm{ADC}$ and EA. All authors have read and agreed to the published version of the manuscript.

Funding The authors have not declared a specific grant for this research from any funding agency in the public, commercial or not-for-profit sectors.

Competing interests None declared.

Patient consent for publication Not required.

Ethics approval The study protocol was approved by the internal Institutional Review Board (ethical approval code: 01/2018). The procedures performed were in accordance with the principles of the 1964 Declaration of Helsinki and its later amendments (2013). Written informed consent was obtained from all study participants before inclusion.

Provenance and peer review Not commissioned; externally peer reviewed.

Data availability statement All data relevant to the study are included in the article or uploaded as supplemental information. All data have been obtained from the authors of this manuscript and are in the possession of the corresponding author.

Open access This is an open access article distributed in accordance with the Creative Commons Attribution Non Commercial (CC BY-NC 4.0) license, which permits others to distribute, remix, adapt, build upon this work non-commercially, and license their derivative works on different terms, provided the original work is properly cited, appropriate credit is given, any changes made indicated, and the use is non-commercial. See: http://creativecommons.org/licenses/by-nc/4.0/.

\section{ORCID iDs}

Emanuele Bisso http://orcid.org/0000-0003-3938-9490

Salvatore Alaimo http://orcid.org/0000-0003-4825-7617

Andrea Di Cataldo http://orcid.org/0000-0002-4509-3066

Maria Salvina Signorelli http://orcid.org/0000-0001-5835-4176

Alfredo Pulvirenti http://orcid.org/0000-0002-9764-0295

Eugenio Aguglia http://orcid.org/0000-0003-2146-7737

\section{REFERENCES}

1 Koohkan E, Yousofian S, Rajabi G, et al. Health information needs of families at childhood cancer: a qualitative study. J Educ Health Promot 2019;8:246.

2 Steliarova-Foucher E, Colombet M, Ries LAG, et al. International incidence of childhood cancer, 2001-10: a population-based registry study. Lancet Oncol 2017;18:719-31. 
3 Curtin SC, Minino AM, Anderson RN. Declines in cancer death rates among children and adolescents in the United States, 1999-2014. NCHS Data Brief 2016;257:1-8.

4 Molina Y, Yi JC, Martinez-Gutierrez J, et al. Resilience among patients across the cancer continuum: diverse perspectives. Clin $J$ Oncol Nurs 2014;18:93-101.

5 Baron M, Tagore M, Hunter MV, et al. The stress-like cancer cell state is a consistent component of tumorigenesis. Cell Syst 2020;11:536-46.

6 Swartzman S, Booth JN, Munro A, et al. Posttraumatic stress disorder after cancer diagnosis in adults: a meta-analysis. Depress Anxiety 2017;34:327-39.

7 Hund B, Reuter K, Härter M, et al. Stressors, symptom profile, and predictors of adjustment disorder in cancer patients. results from an epidemiological study with the composite international diagnostic interview, adaptation for oncology (CIDI-O). Depress Anxiety 2016;33:153-61.

8 Irestorm E, Tonning Olsson I, Johansson B, et al. Cognitive fatigue in relation to depressive symptoms after treatment for childhood cancer. BMC Psychol 2020;8:31.

9 Ernst M, Brähler E, Wild PS, et al. Risk factors for suicidal ideation in a large, registry-based sample of adult long-term childhood cancer survivors. J Affect Disord 2020;265:351-6.

10 Barakat LP, Alderfer MA, Kazak AE. Posttraumatic growth in adolescent survivors of cancer and their mothers and fathers. $J$ Pediatr Psychol 2006;31:413-9.

11 Connor KM. Assessment of resilience in the aftermath of trauma. $J$ Clin Psychiatry 2006;67(Suppl 2):46-9.

12 Mancini AD, Bonanno GA. Predictors and parameters of resilience to loss: toward an individual differences model. J Pers 2009;77:1805-32.

13 Rutter M. Implications of resilience concepts for scientific understanding. Ann N Y Acad Sci 2006;1094:1-12.

14 Seiler A, Jenewein J. Resilience in cancer patients. Front Psychiatry 2019;10:208.

15 Perry JC, Kardos ME, Pagano CJ. The Study of Defenses in Psychotherapy Using the Defense Mechanism Rating Scales (DMRS). In: Hentschel U, Smith GJW, Ehlers W, et al, eds. The Concept of Defense Mechanisms in Contemporary Psychology: Theoretical, Research, and Clinical Perspectives [Internet]. New York NY: Springer New York, 1993: 122-32.

16 Renzi C, Perinel G, Arnaboldi P, et al. Memories of paternal relations are associated with coping and defense mechanisms in breast cancer patients: an observational study. BMC Psychol 2017;5:37.

17 Di Giuseppe M, Ciacchini R, Micheloni T, et al. Defense mechanisms in cancer patients: a systematic review. J Psychosom Res 2018;115:76-86.
18 Gleser GC, Ihilevich D. An objective instrument for measuring defense mechanisms. J Consult Clin Psychol 1969;33:51-60.

19 Cooper C, Kline P. A validation of the defence mechanism inventory. Br J Med Psychol 1982;55 (Pt 3):209-14.

20 Derogatis L. SCL-90-R: Symptom Checklist-90-R:administration, scoring and procedures manual. Minneapolis, MN: National Computer systems, 1994.

21 Schmitz N, Hartkamp N, Kiuse J, et al. The Symptom Check-List-90-R (SCL-90-R): a German validation study. Qual Life Res 2000;9:185-93.

22 Davidson JRT, Book SW, Colket JT, et al. Assessment of a new self-rating scale for post-traumatic stress disorder. Psychol Med 1997;27:153-60.

23 Kevan B. Consistency and factorial invariance of the Davidson trauma scale in heterogeneous populations: results from the 2010 Chilean earthquake. Int J Methods Psychiatr Res 2017;26. doi:10.1002/mpr.1516. [Epub ahead of print: 2507 2016].

24 Hayes AF, Rockwood NJ. Regression-based statistical mediation and moderation analysis in clinical research: observations, recommendations, and implementation. Behav Res Ther 2017;98:39-57.

25 Tingley D, Yamamoto T, Hirose K. mediation: R Package for Causal Mediation Analysis. J Stat Softw 2014;59 mediation: R Package for Causal Mediation Analysis | Tingley | Journal of Statistical Software ( jstatsoft.org)

26 Weihs KL, Enright TM, Simmens SJ, et al. Negative affectivity, restriction of emotions, and site of metastases predict mortality in recurrent breast cancer. J Psychosom Res 2000;49:59-68.

27 Watson M, Haviland JS, Greer S, et al. Influence of psychological response on survival in breast cancer: a population-based cohort study. The Lancet 1999;354:1331-6.

28 Vos MS, de Haes JCJM. Denial in cancer patients, an explorative review. Psychooncology 2007;16:12-25.

29 Goldbeck R. Denial in physical illness. J Psychosom Res 1997;43:575-93.

30 Drapeau M, de Roten Y, Blake E, et al. Defensive flexibility and its relation to symptom severity, depression, and anxiety. J Nerv Ment Dis 2011;199:38-41.

31 Grebot E, Paty B, Girarddephanix N. Relationships between defense mechanisms and coping strategies, facing exam anxiety performance. Encephale 2006;32:315-24.

32 Gianinazzi ME, Rueegg CS, Vetsch J, et al. Cancer's positive FLIP side: posttraumatic growth after childhood cancer. Support Care Cancer 2016;24:195-203.

33 Vaillant GE. Ego mechanisms of defense and personality psychopathology. J Abnorm Psychol 1994;103:44-50.

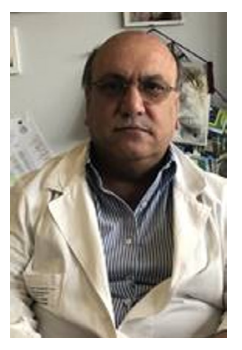

Antonino Petralia graduated in Medicine and Surgery at the University of Catania in 1984, where he obtained PhD in Psychobiology of Mental Disorders with a thesis titled 'Integrated Psychoneuroendocrine and Neuropsychological Approach to the Synchronic and Diachronic Study of Schizophrenia' in 1990, and in 1991, the specialisation in Psychiatry. Since 1990, he has been working at Psychiatric Clinic of the University General Hospital 'G. Rodolico' of Catania, where he currently holds the position of Deputy Director of the Psychiatric Clinic, Head of the 'Ambulatory for mood disorders, anxiety disorders and post-sepsis psychic disorders', Senior Researcher and Professor of Psychiatry in the degree courses of Medicine and Surgery, Nursing, Obstetrics and Psychiatry Rehabilitation Technicians, and Professor in Master for psychiatry and forensic psychology. Given the skills he acquired in the neuropsychopharmacology, he became the councilor and then auditor in the Italian Society of Neuropsychopharmacology, respectively, in 1992-95 and 1995-98. In addition to teaching, he has been involved in research activities, focusing his attention on the deepening of the biological, nosographic, therapeutic and rehabilitative aspects of mental pathology in adults and in developmental age. He has published 2 psychiatry treatises, 7 monographs, and 218 scientific papers in national and international journals. He has participated as Principal Investigator in 45 pharmacological research protocols performed in compliance with the GCP. As a Professor of Master for psychiatry and forensic psychology, for about 25 years, he has collaborated with Judges, Public Prosecutors and Lawyers in the psychiatric-forensic field for the management of psychiatric problems of adults and minors. From 2016 to 2019, he was the President of the Sicilian section of the Italian Society of Psychiatry (S.I.P.). His current main research activities and interests include the correlation between psychopathological dimensions, therapeutic responses and biological aspects. 